\title{
Distribution of atmospheric corrosion grades in Southern Hebei Province
}

\author{
Xiangnan $\mathrm{Niu}^{1}$, Haiping $\mathrm{Hou}^{1}$, Kai $\mathrm{Che}^{1}$, Ning $\mathrm{Yu}^{2}$, Yike $\mathrm{Wei}^{2, *}$, and $Y u \mathrm{Tan}^{2}$ \\ ${ }^{1}$ Electric Power Research Institute of State Grid Hebei Electric Power Co., Ltd, Shijiazhuang, China \\ ${ }^{2}$ North China Electric Power University (Baoding), Hebei, China
}

\begin{abstract}
China has the largest and most complex power grid in the world. But at the same time, the power grid is also facing severe challenges from the natural environment, so its security and reliability are particularly important. In this paper, Q235 carbon steel was used for exposure test. 81 experimental sites were set up in five cities of Southern Hebei Province. And the data of two years were collected and sorted out, and the map of atmospheric corrosion grade in Southern Hebei Province was drawn.

Keywords: Atmospheric corrosion; Regional distribution; Hebei Province; Q235 carbon steel.
\end{abstract}

\section{Introduction}

China has the largest and most complex power grid in the world, with a capacity of more than 1 billion kilowatts and over $800000 \mathrm{~km}$ of UHV transmission lines. But at the same time, the power grid is also facing severe challenges from the natural environment, so its security and reliability are particularly important. Among them, the atmospheric corrosion of power grid equipment is one of the important reasons that restrict its service life and lead to equipment replacement and failure. It has high temperature and high humidity coastal areas and also faces the heavy industry air pollution environment brought by some areas with rapid industrial development. The corrosion law and characteristics of power grid equipment and materials have obvious regional differences. The service life difference caused by different environments is obvious. [1-4]

Since the beginning of the 20th century, foreign countries began to study the atmospheric exposure corrosion of steel, and accumulated a large number of atmospheric corrosion data.[5-8] However, there are few data of similar studies in China. In this study, the standard atmospheric corrosion field test method "exposure" method was used to carry out the coupon sampling work, and the influencing factors of environmental corrosion were collected and tested. The atmospheric corrosion rate and environmental corrosion grade in Southern Hebei Province were obtained by analysing the corrosion rate and influencing factors of the samples after field hanging. Then the map of atmospheric corrosion is drawn.

\footnotetext{
* Corresponding author: wuzhenkee@foxmail.com
} 


\section{Methods}

\subsection{Selection of sample points}

In order to ensure the integrity and accuracy of the atmospheric corrosion map, at least one sampling point is arranged every $1000 \mathrm{~km}^{2}-1500 \mathrm{~km}^{2}$ in the area. Combined with the actual situation, the key points are arranged in the areas with serious corrosion, such as heavy industry area and coastal area.

\subsection{Experiment}

Q235 carbon steel with technical index conforming to GB / T 700 is used for atmospheric environment corrosion test. The size is $150 \mathrm{~mm} \times 70 \mathrm{~mm} \sim 100 \mathrm{~mm} \times 3 \sim 5 \mathrm{~m}$. The samples were pretreated before being put into use. Use cotton yarn and absorbent cotton to wipe the oil on the surface, then use ethanol to clean, and then use $18 \% \mathrm{HCl}+2 \%$ hexamethylenetetramine solution to remove the surface oxide scale. [9] In the pickling process, keep the whole surface pickling uniform. After washing with distilled water, the pickled sample is put into anhydrous alcohol immediately, and then taken out and placed in a dryer for standby after drying. Before the sample is put in, number it and record the original data of each sample, including material, mass and size. The original morphology was photographed and then put into the experiment. Take samples on a scheduled date. After sampling, record and take photos of the samples. Before cleaning, take photos to retain the morphology of the samples after the test. Brush the loose rust on the surface with a bristle brush until it doesn't fall off again, leaving a firm rust layer on the surface. It was then placed in a desiccator for 48 hours and weighed. Then the sample was pickled. The pickled sample was washed with distilled water and immediately put into anhydrous alcohol, then dried and weighed, and the detailed data were recorded. Finally, the corrosion rate was calculated according to Eq. (1). And the experimental flow is shown in Figure 1.

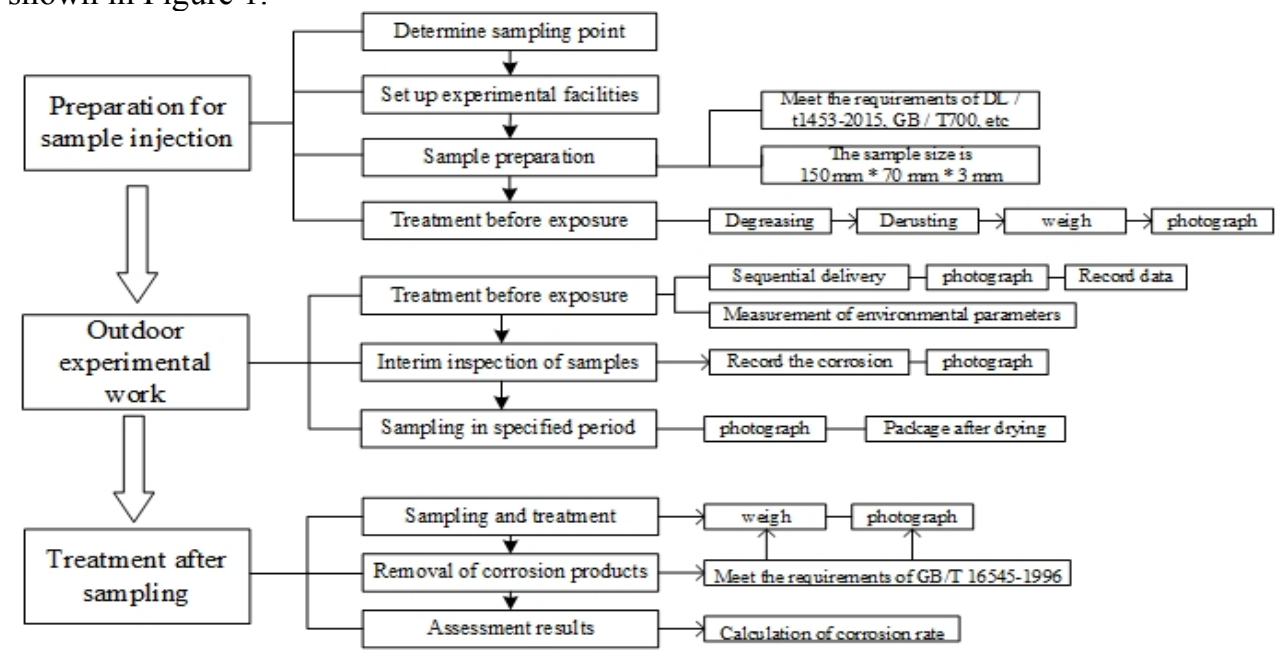

Fig. 1. Process of atmospheric exposure corrosion test.

$$
V=3.65 \times 10^{3} \times\left(m_{0}-m_{1}\right) /(S \times T \times \rho)
$$

Where $V$ is the corrosion rate, $m_{0}$ is the weight of sample before experiment, $m_{l}$ is the weight of sample after experiment, $S$ is the total area of specimen, $T$ is the temperature, $\rho$ is the sample density. 


\section{Result}

According to ISO 9223-2012 [10], the atmospheric corrosivity is divided into six grades from $\mathrm{C}_{1}$ to $\mathrm{C}_{\mathrm{x}}$. The Atmospheric Corrosivity in the study area is determined by the corrosion loss in the first year. The first-year corrosion rate of carbon steel and the corresponding corrosion grade of corrosion rate are listed in Tab. 1.

Table 1. Classification standard for atmospheric corrosivity.

\begin{tabular}{ccc}
\hline Atmospheric corrosion grade & $\begin{array}{c}\text { Corrosion rate of carbon } \\
\text { steel }(\mu \mathrm{m} / \mathrm{a})\end{array}$ & corrosive \\
\hline $\mathrm{C}_{1}$ & $\mathrm{~V} \leq 1.3$ & Very low \\
$\mathrm{C}_{2}$ & $1.3<\mathrm{V} \leq 25$ & Low \\
$\mathrm{C}_{3}$ & $25<\mathrm{V} \leq 50$ & Medium \\
$\mathrm{C}_{4}$ & $50<\mathrm{V} \leq 80$ & High \\
$\mathrm{C}_{5}$ & $80<\mathrm{V} \leq 200$ & Very high \\
$\mathrm{C}_{\mathrm{x}}$ & $200<\mathrm{V} \leq 700$ & Extreme \\
\hline
\end{tabular}

According to the average atmospheric corrosion rate data of the first year, the atmospheric corrosion map of Southern Hebei is drawn, as shown in Figure 2. It can be seen that the areas with higher atmospheric corrosion level are mainly concentrated in Handan. Handan is rich in mineral resources. Relying on mineral resources, Handan has developed more steel and thermal power industries, and is one of the important industrial cities in Hebei Province. The development of industry also brings about the atmospheric corrosion problem in the first degree, which leads to the higher degree of atmospheric corrosion than other cities in Hebei. Figure 3 shows the atmospheric corrosion map generated by the average corrosion rate in the second year. It can be seen that the atmospheric corrosion degree of Handan test site is relatively high. In addition, it is worth noting that as an important sea port in the south of Hebei Province, Cangzhou coastal area has also developed a certain scale of industry. Under the influence of coastal areas and industries, it can be seen that coastal areas also have serious atmospheric corrosion to a certain extent.

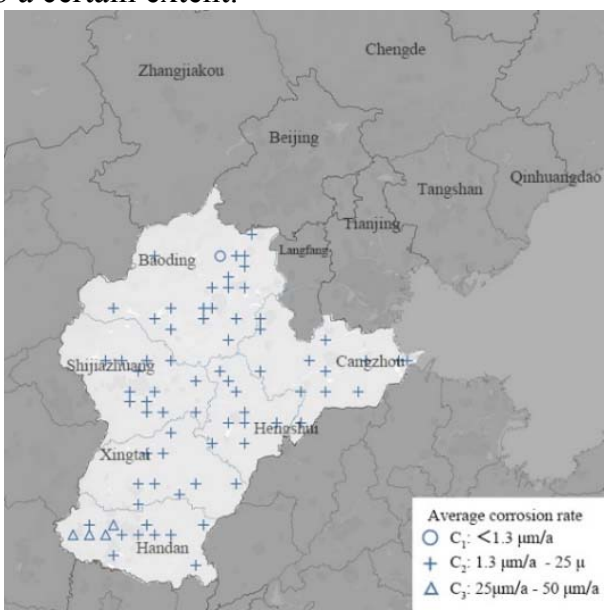

Fig. 2. Distribution map of atmospheric corrosion test sites and corrosion grades in Southern Hebei Province(2019). 


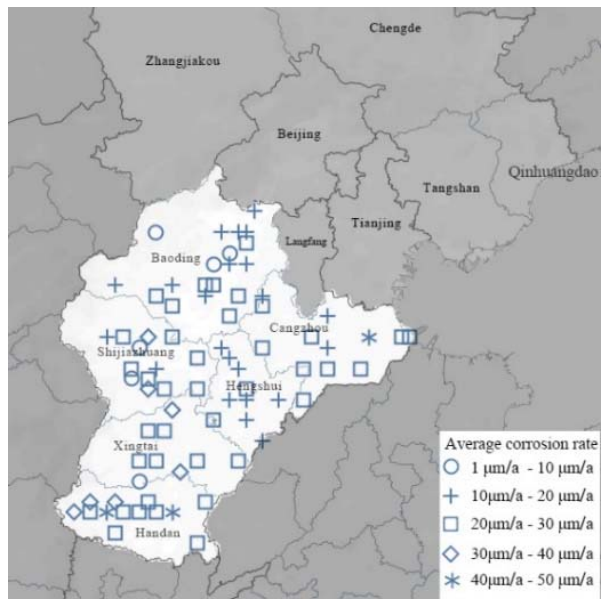

Fig. 3. Atmospheric corrosion test points and corrosion conditions in Southern Hebei Province(2020).

\section{Conclusion}

The study is based on the research background of atmospheric corrosion of transmission towers in five cities of Southern Hebei Province of State Grid, the atmospheric corrosion of Shijiazhuang, Baoding, Hengshui, Xingtai, Handan and Cangzhou in Hebei Province was evaluated by exposure method using Q235 carbon steel. Based on the analysis of the data collected from 81 test sites in the past two years, the atmospheric corrosion level is in the middle and low level as a whole. In Handan, Shijiazhuang and other heavy industry intensive areas, as well as coastal areas of Cangzhou, the atmospheric corrosion degree is higher than other areas.

Atmospheric corrosion has many effects on the corrosion of materials. This paper can provide some reference for the protection of local metal materials, but more in-depth research is needed.

\section{References}

1. Singh D. N., Yadav S., Saha J.K.. Role of climatic conditions on corrosion characteristics of structural steels [J]. Corrosion Science, 2008, 50(1): 93-110

2. Corvo F., Minotas J., Delgado J., et al. Changes in atmospheric corrosion rate caused by chloride ions depending on rain regime[J]. Corrosion Science, 2005, 47(4): 883-892

3. Corvo F., Haces C., Betancourt N., et al. Atmospheric corrosivity in the Caribbean area[J]. Corrosion Science, 1997, 39 (5): 823-833

4. Lu Z., Macdonald D. D.. Transient growth and thinning of the barrier oxide layer on iron measured by real-time spectroscopic ellipsometry[J]. Electrochemical Acta, 2008, 53(26): 7696-7702

5. Mendoza A. R., Corvo F.. Outdoor and indoor atmospheric corrosion of carbon steel[J]. Corrosion Science, 1999, 41(1):75-86

6. Castaño J. G., Botero C. A., Restrepo A. H., et al. Atmospheric corrosion of carbon steel in Colombia[J]. Corrosion Science, 2010, 52(1): 216-223

7. Morales J., Martín-Krijer S., Díaz F., et al. Atmospheric corrosion in subtropical areas: influences of time of wetness and deficiency of the ISO 9223 norm[J]. Corrosion Science, 2005, 47(8): 2005-2019 
8. Syed S.. Atmospheric corrosion of hot and cold rolled carbon steel under field exposure in Saudi Arabia[J]. Corrosion Science, 2008, 50(6): 1779-1784

9. GBT 16545-2015 Corrosion of metals and alloys removal of corrosion products from corrosion specimens

10. International Organization for Standardization. ISO 9223: 2012.Corrosion of metals and alloys, corrosivity of atmospheres, classification, determination and estimation[S]. Switzerland: 2012 